\title{
Macrofungal biodiversity of Gürpınar (Van) district
}

\section{Ant J Bot aty}

Received : 24.12 .2020 Accepted : 19.01.2021 Online : 24.01 .2021

Ezelhan ŞELEM ${ }^{1} \odot$, Ali KELEŞ ${ }^{2 *}$, Sinan IŞSER ${ }^{3} \bullet$

${ }^{1}$ Yüzüncü Yll University, Agriculture Faculty, Department of Field Crops, Van, Turkey

${ }^{2,3}{ }^{3}$ üzüncü Yll University, Education Faculty, Department of Science and Mathematics Education, Van, Turkey alikeles61@yahoo.com, ${ }^{1}$ ezelhanselem@hotmail.com, ${ }^{3}$ sinan_1970@hotmail.com

\section{Gürpınar (Van) yöresinin makromantar biyoçeşitliliği}

Abstract: The study was based on macrofungi samples collected from Gürpinar district of Van province between 2015 and 2017. As a result of field and laboratory studies 94 macrofungi species belonging to 49 genera, 27 families, seven orders and three classes within Ascomycota and Basidiomycota were determined. The list of the determined taxa were presented together with their habitats, substrates, collection localities and personel voucher numbers.

Key words: Biodiversity, macrofungi, mycota, Turkey

Özet: Çalışma 2015 ve 2017 yıllarında Van'ın Gürpınar ilçesinden toplanan makromantar örnekleri üzerinde gerçekleștirilmiştir. Arazi ve laboratuvar çalışmaları sonucunda Ascomycota ve Basidiomycota bölümleri içinde yer alan üç sınıf, yedi takım, 27 familya ve 49 cinse ait 94 tür belirlenmiştir. Belirlenen taksonlar, habitatları, substratları, toplanma yerleri ve toplayıcı numaraları ile birlikte listelenmiştir.

Anahtar Kelimeler: Biyoçeşitlilik, makromantar, mikota, Türkiye

Citation: Şelem E, Keleş A, İşler S (2021). Macrofungal biodiversity of Gürpınar (Van) district. Anatolian Journal of Botany 5(1): 2328.

\section{Introduction}

The kingdom Fungi constitutes the second most diverse living group in the world with about more than 1.5 million species (Hawksworth et al., 1995). They can grow almost everywhere in the world as saprophytes, parasites and symbionts, and those with fruiting bodies that can be seen by naked eye are known as macrofungi. Besides being consumed as food, macrofungi are used in cosmetics and pharmacology and have high economic value (Adanacıŏlu et al., 2016; Süfer et al., 2016).

Studies on the macrofungal biodiversity of Turkey have been started in the first quarter of the $19^{\text {th }}$ century, and lots of works have been conducted by many researchers (Alkan et al., 2010; Demirel et al., 2010; Doğan et al., 2012; Türkekul and Işık, 2016; Uzun et al., 2017; Yıldız et al., 2019; Acar et al., 2020). Sesli and Denchev (2014) present about 2.400 macrofungi species in Turkey, and Kaya and Uzun (2018) introduces this number as 2.500. Considering, the 15.000 macromycete taxa (Lukić, 2008) determined in Europe, it becomes clear that there is a lot of work to be done in Turkey.

Gürpınar is the largest district of Turkey with a surface area of $4.063 \mathrm{~km}^{2}$ within the boundaries of Van province (Fig. 1 ), and located between $37^{\circ} 44^{\prime}-28^{\circ} 29^{\prime}$ north latitudes and $43^{\circ} 07^{\prime}-44^{\circ} 07^{\prime}$ east longitudes. The district lies within the IranoTuranean phytogeographical flora sector. The climate of the research area is Mediterranean with an annual rainfall of $281 \mathrm{~mm}$ and an annual average temperature of $8.1{ }^{\circ} \mathrm{C}$ (Bani and Adıgüzel, 2008). Though the list of naturally growing edible mushrooms was presented by Şelem et al. (2019), there isn't a detailed study on the overall macrofungal biodiversity of Gürpınar district.

The study aims to determine naturally growing macrofungi of the district and make a contribution to the mycobiota of Turkey.

\section{Materials and Method}

Macrofungi samples were collected from the region within the boundaries of Gürpınar districts of Van province. During field studies, first of all the fruit bodies were photographed at their natural habitats. Then necessary notes about the ecological and morphological characteristics and the geographical positions of the the samples were recorded. The collected samples were in paper boxes and transferred to the fungarium. They were dried in an air conditioned room and kept as fungarium materials in polyethylene bags. Further investigations were carried out in the fungarium on dried samples. Microscopic investigations were performed under a compound microscope. The specimens were identified by comparing the obtained data with the relevant literature (Moser, 1983; Breitenbach and Kränzlin, 1984, 1986, 1991, 1995, 2000; Buczacki, 1989; Bresinsky and Besl, 1990; Jordan, 1995; Pegler et al., 1995; Philips, 1991; Dähncke, 2004; Hausknecht, 2009; Uzun, 2010; Kuo and Methven, 2014). The determined macrofungi samples are kept in the fungarium of Biology Department, Science Faculty, Van Yüzüncü Y1l Üniversity(VANF).

\section{Results}

The determined taxa are listed in alphabetical order. Index Fungorum (accessed on 20 December 2020) were followed for the systematics of taxa. Previously reported taxa were given with the citation.

\section{Ascomycota Whittaker}

Leotiomycetes O.E. Erikss. \& Winka

Helotiales Nannf.

Helotiaceae Rehm

1. Hymenoscyphus calyculus (Fr.) W. Phillips: On decaying Populus sp., twigs, locality 17, 05.10.2016, Şelem 276. 


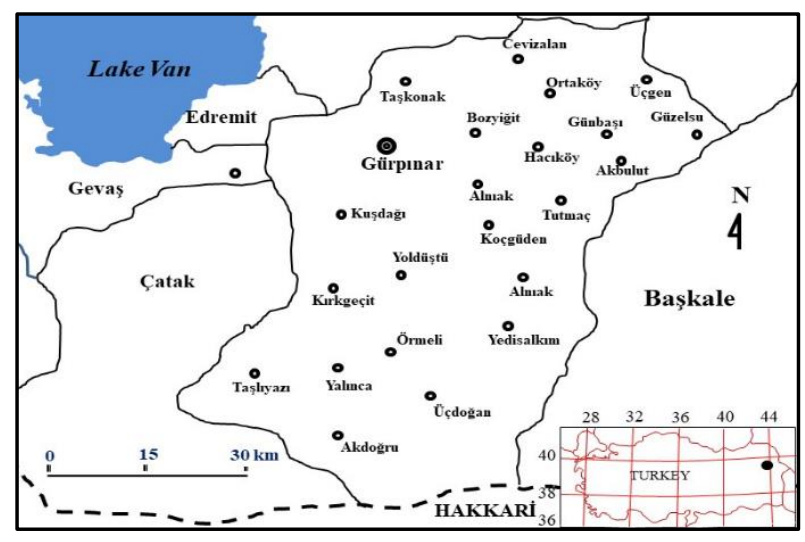

Figure 1. Map of the research area
Lachnaceae Raitv.

2. Lachnum bicolor (Bull.) P. Karst.: On dead Salix sp. branches, locality 17, 05.10.2016, Şelem 263.

Pezizomycetes O.E.Erikss. \& Winka

Pezizales J.Schröt.

Helvellaceae Fr.

3. Helvella acetabulum (L.) Quél: (Şelem et al., 2019).

4. Helvella crispa (Scop.) Fr.: On soil under Populus sp., locality 17, 05.10.2016, Şelem 276.

5. Helvella lacunosa Afzel.: (Şelem et al., 2019).

6. Helvella leucopus Pers.: (Şelem et al., 2019).

7. Paxina queletii (Bres.) Stangl: (Şelem et al., 2019).

Table 1. Collection localities of the macrofungi samples

\begin{tabular}{|c|c|c|c|}
\hline Loc. No & Locality & Coordinates & Altitude (m) \\
\hline 1 & Akbulut village & $38^{\circ} 18^{\prime} 06^{\prime \prime} \mathrm{N} ; 43^{\circ} 41^{\prime} 02^{\prime \prime} \mathrm{E}$ & 2186 \\
\hline 2 & Albenek village & $38^{\circ} 36^{\prime} 01^{\prime \prime} \mathrm{N} ; 43^{\circ} 17^{\prime} 01^{\prime \prime} \mathrm{E}$ & 2022 \\
\hline 3 & Alniak village & $38^{\circ} 13^{\prime} 12^{\prime \prime} \mathrm{N} ; 43^{\circ} 42^{\prime} 48^{\prime \prime} \mathrm{E}$ & 2502 \\
\hline 4 & Bozyiğit Village & $38^{\circ} 22^{\prime} 57^{\prime \prime} \mathrm{N} ; 43^{\circ} 34^{\prime} 08^{\prime \prime} \mathrm{E}$ & 1845 \\
\hline 5 & Cevizalan village & $38^{\circ} 24^{\prime} 19^{\prime \prime} \mathrm{N} ; 43^{\circ} 47^{\prime} 56^{\prime \prime} \mathrm{E}$ & 2172 \\
\hline 6 & Çörekli village & $38^{\circ} 21^{\prime} 53^{\prime \prime} \mathrm{N} ; 43^{\circ} 47^{\prime} 54^{\prime \prime} \mathrm{E}$ & 2001 \\
\hline 7 & Cevizalan village & $38^{\circ} 22^{\prime} 16^{\prime \prime} \mathrm{N} ; 43^{\circ} 47^{\prime} 34^{\prime \prime} \mathrm{E}$ & 2025 \\
\hline 8 & Erkaldı quarter & $38^{\circ} 21^{\prime} 26^{\prime \prime} \mathrm{N} ; 43^{\circ} 33^{\prime} 04^{\prime \prime} \mathrm{E}$ & 1809 \\
\hline 9 & Giyimli village & $38^{\circ} 12^{\prime} 16^{\prime \prime} \mathrm{N} ; 43^{\circ} 47^{\prime} 05^{\prime \prime} \mathrm{E}$ & 2322 \\
\hline 10 & Günbaşı village & $38^{\circ} 18^{\prime} 46^{\prime \prime} \mathrm{N} ; 43^{\circ} 43^{\prime} 45^{\prime \prime} \mathrm{E}$ & 2168 \\
\hline 11 & Gürpınar (entrance) & $38^{\circ} 19^{\prime} 27^{\prime \prime} \mathrm{N} ; 43^{\circ} 24^{\prime} 06^{\prime \prime} \mathrm{E}$ & 1745 \\
\hline 12 & Gürpınar centre & $38^{\circ} 19^{\prime} 33^{\prime \prime} \mathrm{N} ; 43^{\circ} 24^{\prime} 24^{\prime \prime} \mathrm{E}$ & 1751 \\
\hline 13 & Gürpınar-Hakkari highway 14th km & $38^{\circ} 21^{\prime} 59^{\prime \prime} \mathrm{N} ; 43^{\circ} 34^{\prime} 02^{\prime \prime} \mathrm{E}$ & 2010 \\
\hline 14 & Güzelsu village & $38^{\circ} 18^{\prime} 58^{\prime \prime} \mathrm{N} ; 43^{\circ} 48^{\prime} 04^{\prime \prime} \mathrm{E}$ & 1982 \\
\hline 15 & Güzelsu village & $38^{\circ} 18^{\prime} 52^{\prime \prime} \mathrm{N} ; 43^{\circ} 48^{\prime} 25^{\prime \prime} \mathrm{E}$ & 1980 \\
\hline 16 & Güzelsu village & $38^{\circ} 19^{\prime} 11^{\prime \prime} \mathrm{N} ; 43^{\circ} 48^{\prime} 07^{\prime \prime} \mathrm{E}$ & 1996 \\
\hline 17 & Güzelsu village & $38^{\circ} 19^{\prime} 00^{\prime \prime} \mathrm{N} ; 43^{\circ} 47^{\prime} 58^{\prime \prime} \mathrm{E}$ & 1977 \\
\hline 18 & Güzelsu village & $38^{\circ} 19^{\prime} 02^{\prime \prime} \mathrm{N} ; 43^{\circ} 48^{\prime} 12^{\prime \prime} \mathrm{E}$ & 1986 \\
\hline 19 & Güzelsu village & $38^{\circ} 18^{\prime} 57^{\prime \prime} \mathrm{N} ; 43^{\circ} 48^{\prime} 00^{\prime \prime} \mathrm{E}$ & 1972 \\
\hline 20 & Hacıköy village & $38^{\circ} 18^{\prime} 22^{\prime \prime} \mathrm{N} ; 43^{\circ} 38^{\prime} 23^{\prime \prime} \mathrm{E}$ & 2205 \\
\hline 21 & Hamurkesen village & $38^{\circ} 20^{\prime} 38^{\prime \prime} \mathrm{N} ; 43^{\circ} 37^{\prime} 29^{\prime \prime} \mathrm{E}$ & 1948 \\
\hline 22 & Işıkpınar village & $38^{\circ} 19^{\prime} 16^{\prime \prime} \mathrm{N} ; 43^{\circ} 37^{\prime} 01^{\prime \prime} \mathrm{E}$ & 2134 \\
\hline 23 & Kırgeçit village & $38^{\circ} 08^{\prime} 17^{\prime \prime} \mathrm{N} ; 43^{\circ} 29^{\prime} 43^{\prime \prime} \mathrm{E}$ & 2105 \\
\hline 24 & Kırgeçit village & $38^{\circ} 08^{\prime} 35^{\prime \prime} \mathrm{N} ; 43^{\circ} 31^{\prime} 27^{\prime \prime} \mathrm{E}$ & 2147 \\
\hline 25 & Kırgeçit village & $38^{\circ} 08^{\prime} 08^{\prime \prime} \mathrm{N} ; 43^{\circ} 31^{\prime} 17^{\prime \prime} \mathrm{E}$ & 2165 \\
\hline 26 & Koçgüden village & $38^{\circ} 09^{\prime} 20^{\prime \prime} \mathrm{N} ; 43^{\circ} 38^{\prime} 33^{\prime \prime} \mathrm{E}$ & 2570 \\
\hline 27 & Kuş̧ağ1 village & $38^{\circ} 14^{\prime} 35^{\prime \prime} \mathrm{N} ; 43^{\circ} 27^{\prime} 29^{\prime \prime} \mathrm{E}$ & 1905 \\
\hline 28 & Muratald1 village & $38^{\circ} 15^{\prime} 17^{\prime \prime} \mathrm{N} ; 43^{\circ} 51^{\prime} 08^{\prime \prime} \mathrm{E}$ & 2136 \\
\hline 29 & Ortaköy village & $38^{\circ} 22^{\prime} 02^{\prime \prime} \mathrm{N} ; 43^{\circ} 38^{\prime} 04^{\prime \prime} \mathrm{E}$ & 1910 \\
\hline 30 & Ortaköy village & $38^{\circ} 21^{\prime} 50^{\prime \prime} \mathrm{N} ; 43^{\circ} 37^{\prime} 55^{\prime \prime} \mathrm{E}$ & 1868 \\
\hline 31 & Örmeli village & $38^{\circ} 07^{\prime} 05^{\prime \prime} \mathrm{N} ; 43^{\circ} 30^{\prime} 52^{\prime \prime} \mathrm{E}$ & 2231 \\
\hline 32 & Sapakonak village & $38^{\circ} 12^{\prime} 46^{\prime \prime} \mathrm{N} ; 43^{\circ} 36^{\prime} 35^{\prime \prime} \mathrm{E}$ & 2537 \\
\hline 33 & Sevindik village & $38^{\circ} 18^{\prime} 11^{\prime \prime N} ; 43^{\circ} 52^{\prime} 51^{\prime \prime} \mathrm{E}$ & 2098 \\
\hline 34 & Sevindik village & $38^{\circ} 18^{\prime} 22^{\prime \prime} \mathrm{N} ; 43^{\circ} 52^{\prime} 27^{\prime \prime} \mathrm{E}$ & 2038 \\
\hline 35 & Taşdöndüren village & $38^{\circ} 16^{\prime} 01^{\prime \prime} \mathrm{N} ; 43^{\circ} 48^{\prime} 53^{\prime \prime} \mathrm{E}$ & 2031 \\
\hline 36 & Tepegören village & $38^{\circ} 21^{\prime} 35^{\prime \prime} \mathrm{N} ; 43^{\circ} 53^{\prime} 13^{\prime \prime} \mathrm{E}$ & 2107 \\
\hline 37 & Tutmaç village & $38^{\circ} 15^{\prime} 08^{\prime \prime} \mathrm{N} ; 43^{\circ} 42^{\prime} 34^{\prime \prime} \mathrm{E}$ & 2401 \\
\hline 38 & Üçgen village & $38^{\circ} 22^{\prime} 31^{\prime \prime} \mathrm{N} ; 43^{\circ} 44^{\prime} 46^{\prime \prime} \mathrm{E}$ & 2141 \\
\hline
\end{tabular}




\begin{tabular}{llll}
\hline 39 & Üçgen village & $38^{\circ} 23^{\prime} 05^{\prime \prime} \mathrm{N} ; 43^{\circ} 44^{\prime} 34^{\prime \prime} \mathrm{E}$ & 2135 \\
40 & Yedisalkım village & $38^{\circ} 11^{\prime} 08^{\prime \prime} \mathrm{N} ; 43^{\circ} 42^{\prime} 11^{\prime \prime} \mathrm{E}$ & 2441 \\
41 & Yoldüştü village & $38^{\circ} 09^{\prime} 15^{\prime \prime} \mathrm{N} ; 43^{\circ} 33^{\prime} 12^{\prime \prime} \mathrm{E}$ & 2187 \\
42 & Yurtbaş1 village & $38^{\circ} 14^{\prime} 08^{\prime \prime} \mathrm{N} ; 43^{\circ} 47^{\prime} 59^{\prime \prime} \mathrm{E}$ & 2108 \\
43 & Zernek village & $38^{\circ} 21^{\prime} 26^{\prime \prime} \mathrm{N} ; 43^{\circ} 39^{\prime} 25^{\prime \prime} \mathrm{E}$ & 1934 \\
44 & Zernek village & $38^{\circ} 21^{\prime} 24^{\prime \prime} \mathrm{N} ; 43^{\circ} 39^{\prime} 26^{\prime \prime} \mathrm{E}$ & 1900 \\
\hline
\end{tabular}

Morchellaceae Rchb.

8. Mitrophora semilibera (DC.) Lév.: (Şelem et al., 2019).

9. Morchella elata Fr.: (Şelem et al., 2019).

10. Morchella esculenta (L.) Pers.: (Şelem et al., 2019).

11. Morchella esculentoides M. Kuo, Dewsbury, Moncalvo \& S.L. Stephenson: (Şelem et al., 2019).

12. Morchella prava Dewsbury, Moncalvo, J.D. Moore \& M. Kuo.: (Şelem et al., 2019).

13. Verpa conica (O.F. Müll.) Sw.: On soil under Populus and Salix sp., locality 13, 03.06.2016, Şelem 34.

14. Geopora arenicola (Lév.) Kers: In soil under Populus sp., locality 12, 05.10.2016, Şelem 101; locality 15, 05.10.2016, Şelem 310; locality 23, 03.06.2016, Şelem 254.

15. Geopora arenosa (Fuckel) S. Ahmad: On soil under Populus sp., locality 16, 18.05.2015, Şelem 23.

16. Geopora sepulta (Fr.) Korf \& Burds.: On soil under Populus sp., locality 29, 18.05.2015, Şelem 74.

17. Pulvinula convexella (P. Karst.) Pfister.: On burned ground, locality 15, 05.10.2016, Şelem 289.

18. Scutellinia scutellata (L.) Lambotte: On damp soil among leaf litter, locality 15, 05.10.2016, Şelem 290.

19. Tricharina praecox (P. Karst.) Dennis: On damp soil, locality 17, 05.10.2016, Şelem 273.

20. Trichophaea pseudogregaria (Rick) Boud.: (Keleş and Şelem, 2017).

Pezizaceae Dumort.

21. Peziza succosa Berk.: On burned ground, locality 18, 18.05.2015, Şelem 30.

Basidiomycota R.T.Moore

Agaricomycetes Doweld

Agaricales Underw.

Agaricaceae Chevall.

22. Agaricus bisporus (J.E. Lange) Imbach: (Şelem et al., 2019).

23. Agaricus campestris L.: (Şelem et al., 2019).

24.Agaricus urinascens (Jul. Schäff. \& F.H. Møller) Singer: (Şelem et al., 2019).

25. Bovista plumbea Pers.: (Şelem et al., 2019).

26. Coprinus comatus (O.F. Müll.) Pers.: (Şelem et al., 2019).

27. Cyathus olla (Batsch) Pers.: On soil near woody debris, locality 17, 18.05.2015, Şelem 59.

28. Lepiota cristata (Bolton) P. Kumm: On soil among leaf litter, locality 8, 05.06.2015, Şelem 171.
29. Lepiota subincarnata J.E. Lange: On soil under Salix sp., locality 15, 05.10.2016, Şelem 299.

\section{Bolbitiaceae Singer}

30. Conocybe aporos Kits van Wav.: On soil under Salix sp., locality 17, 05.06.2015, Şelem 137.

31. Conocybe fuscimarginata (Murrill) Singer: On soil under Salix sp., locality 14, 18.05.2015, Şelem 43; locality 17, 05.06.2015, Şelem 145.

32. Conocybe pygmaeoaffinis (Fr.) Kühner: On soil under Salix sp., locality 17, 05.06.2015, Şelem 146.

33. Conocybe rickeniana P.D. Orton: On soil among grass, locality 17, 05.06.2015, Şelem 149.

34. Conocybe tenera (Schaeff.) Fayod: On soil among grass, locality 17,18.05.2015, Şelem 43.

35. Conocybe vestita (Fr.) Kühner: On soil among grass, locality 11, 18.05.2015, Şelem 27.

Cortinariaceae R. Heim ex Pouzar

36. Cortinarius decipiens (Pers.) Fr.: On soil under Salix sp., locality 8, 05.06.2015, Şelem 175.

37. Cortinarius vernus $\mathrm{H}$. Lindstr. \& Melot: On soil under Populus sp., locality 15, 05.10.2016, Şelem 297.

\section{Cyphellaceae Lotsy}

38. Chondrostereum purpureum (Pers.) Pouzar: On Populus sp. stump, locality 11, 18.11.2015, Şelem 297; locality 8, 05.06.2015, Şelem 167.

\section{Entolomataceae Kotl. \& Pouzar}

39. Entoloma caccabus (Kühner) Noordel.: On soil under Salix sp., locality 11, 19.05.2016, Şelem 343.

40. Entoloma rusticoides (Gillet) Noordel: On soil under Malus sp., locality 11, 18.05.2015, Şelem 108.

Hymenogastraceae Vittad.

41. Hebeloma mesophaeum (Pers.) Quél.: On soil under Salix sp., locality 17, 05.06.2015, Şelem 139.

42. Hebeloma pusillum J.E. Lange: On soil under Salix sp., locality 17, 18.05.2015, Şelem 53; on soil under Populus sp., locality 17, 05.10.2016, Şelem 256 .

43. Hypholoma fasciculare (Huds.) P. Kumm.: On Populus sp. stump, locality 17, 05.06.2015, Şelem 165.

44. Psilocybe coronilla (Bull.) Noordel.: (Şelem et al., 2019).

\section{Incertae sedis}

45. Panaeolus fimicola (Pers.) Gillet: On decaying cow dung, locality 4, 18.11.2015, Şelem 201.

Inocybaceae Jülich

46. Crepidotus vulgaris Hesler \& A.H. Sm.: On decaying Salix sp. stump, locality 13, 03.06.2016, Şelem 222. 
47. Inosperma maculatum (Boud.) Matheny \& EsteveRav.: On soil under Salix sp., locality 28, 08.11.2015, Şelem 280.

48. Inocybe cincinnata (Fr.) Quél.: On soil under Populus sp., locality 30, 18.06.2016, Şelem 79.

49. Inocybe dulcamara (Pers.) P. Kumm.: On soil under Populus sp., locality 13, 18.05.2015, Şelem 81; on soil under Salix sp., locality 14, 05.06.2015, Şelem 119; locality 17, 18.11.2015, Şelem 235.

50. Inocybe flocculosa Sacc.: On soil under Populus sp., locality 14, 18.05.2015, Şelem 37.

51. Inocybe fuscomarginata Kühner: On soil under Salix sp., locality 17, 05.06.2015, Şelem 145 .

52. Inocybe perbrevis (Weinm.) Gillet: On soil under Salix sp., locality 17, 05.10.2016, Şelem 121; on soil under Populus sp., locality 16, 05.06.2015, Şelem 278.

53. Pseudosperma rimosum (Bull.) Matheny \& EsteveRav.: On soil under Populus sp., locality 8, 05.06.2015, Şelem 169; on soil under Salix sp., locality 4, 18.11.2015, Şelem 197.

\section{Pleurotaceae Kühner}

54. Pleurotus eryngii (DC.) Quél.: (Şelem et al., 2019).

55. Pleurotus ostreatus (Jacq.) P. Kumm.: (Şelem et al., 2019).

56. Pleurotus populinus O. Hilber \& O.K. Mill.: (Şelem et al., 2019).

\section{Pluteaceae Kotl. \& Pouzar}

57. Pluteus aurantiorugosus (Trog) Sacc.: (Şelem et al., 2019).

58. Pluteus romellii (Britzelm.) Sacc.: (Şelem et al., 2019).

59. Volvopluteus gloiocephalus (DC.) Vizzini, Contu \& Justo: (Şelem et al., 2019).

Psathyrellaceae Vilgalys, Moncalvo \& Redhead

60. Coprinellus disseminatus (Pers.) J.E. Lange: (Şelem et al., 2019).

61. Coprinellus domesticus (Bolton) Vilgalys, Hopple \& Jacq. Johnson: On soil around Populus sp. stump, locality 44, 18.05.2015, Şelem 68.

62. Coprinellus micaceus (Bull.) Vilgalys, Hopple \& Jacq. Johnson: (Şelem et al., 2019).

63. Coprinopsis acuminata (Romagn.) Redhead, Vilgalys \& Moncalvo: On soil aronud Populus sp. stump, locality 12, 18.11.2015, Şelem 213.

64. Coprinopsis atramentaria (Bull.) Redhead, Vilgalys \& Moncalvo: (Şelem et al., 2019).

65. Parasola auricoma (Pat.) Redhead, Vilgalys \& Hopple: On soil among grass, locality 17, 05.06.2015, Şelem 164.

66. Parasola hemerobia (Fr.) Redhead, Vilgalys \& Hopple: On soil under Salix sp., locality 17, 05.06.2015, Şelem 152.

67. Parasola kuehneri (Uljé \& Bas) Redhead, Vilgalys \& Hopple: On soil under Salix sp., locality0 17, 05.06.2015, Şelem 138.

68. Parasola lactea (A.H. Sm.) Redhead, Vilgalys \& Hopple: On soil under Salix sp., locality 17, 05.06.2015, Şelem 142.
69. Parasola plicatilis (Curtis) Redhead, Vilgalys \& Hopple: On soil among grass, locality 15, 05.10.2016, Şelem 294.

70. Psathyrella candolleana (Fr.) Maire: (Şelem et al., 2019).

71. Psathyrella fatua (Fr.) P. Kumm. Populus sp. ağaçları alt1, 17, 18.05.2015, Şelem 56.

72. Psathyrella panaeoloides (Maire) Arnolds: On soil under Salix sp., locality 17, 05.06.2015, Şelem 162; locality 15, 05.10.2016, Şelem 302.

73. Psathyrella potteri A.H. Sm.: On soil under Populus and Salix sp., locality 13, 18.05.2015, Şelem 103.

74. Psathyrella prona (Fr.) Gillet: On soil under Populus and Salix sp., locality 13, 18.05.2015, Şelem 204; locality 17, 05.10.2015, Şelem 263; locality 15, 05.10.2016, Şelem 301.

75. Psathyrella pseudogracilis (Romagn.) M.M. Moser: On decaying stump, locality 17, 18.05.2015. Şelem 58.

Strophariaceae Singer \& A.H. Sm.

76. Agrocybe dura (Bolton) Singer: (Şelem et al., 2019).

77. Agrocybe paludosa (J.E. Lange) Kühner \& Romagn. ex Bon: On soil among grass, locality 17, 05.06.2015 .Şelem 148.

78. Agrocybe pediades (Fr.) Fayod: (Şelem et al., 2019).

79. Agrocybe praecox (Pers.) Fayod: (Şelem et al., 2019).

80. Cyclocybe cylindracea (DC.) Vizzini \& Angelini: (Şelem et al., 2019). On Populus sp. stump, locality 13, 05.10.2016, Şelem 268.

81. Pholiota aurivella (Batsch) P. Kumm.: (Şelem et al., 2019).

Tricholomataceae Lotsy

82. Melanoleuca angelesiana A.H. Sm.: On soil among needle litter under Pinus sp., locality 29, 05.06.2015, Şelem 179.

83. Melanoleuca brevipes (Bull.) Pat.: (Şelem et al., 2019).

84. Melanoleuca cognata (Fr.) Konrad \& Maubl.: (Şelem et al., 2019).

85. Pseudoclitocybe cyathiformis (Bull.) Singer: (Şelem et al., 2019).

86. Lepista personata (Fr.) Cooke: (Şelem et al., 2019).

Tubariaceae Vizzini

87. Tubaria conspersa (Pers.) Fayod: On damp soil among leaf litter under Salix sp., 17, 05.06.2015, Şelem 122.

88. Tubaria furfuracea (Pers.) Gillet: On woody debris, locality 30, 18.11.2015, Şelem 195

Boletales E.-J. Girbert

Suillaceae Besl \& Bresinsky

89. Suillus collinitus (Fr.) Kuntze: (Şelem et al., 2019).

Hymenochaetales Oberw.

Hymenochaetaceae Donk

90. Phellinus igniarius (L.) Quél.: On Malus sp. stump, locality 29, 18.11.2015, Şelem 189.

Polyporales Gäum.

Polyporaceae Fr. ex Corda 
91. Fomes fomentarius (L.) Fr.: On Populus sp. stump, locality 20, 28.06.2017, Şelem 41.

92. Cerioporus squamosus (Huds.) Quél.: (Şelem et al., 2019).

93. Trametes trogii Berk.: On Populus sp. stump, locality 17, 05.06.2015, Şelem 118; locality 18, 19.05.2016, Şelem 332; locality 26, 13.06.2017, Şelem 375; locality 5, 13.11.2016, Şelem 381.

94. Trametes versicolor (L.) Lloyd: On Populus sp. stump, locality 19, 19.05.2016, Şelem 362.

\section{Discussions}

Ninety four macrofungi species belonging to 49 genera, 27 families, seven orders and three classes were determined from Gürpınar district. Twenty one of the determined taxa belong to Ascomycota (Leotiomycetes 2, Pezizomycetes 19) while 73 belong to Basidiomycota (Agaricomycetes 73). Except previously reported 36 edible species (Şelem et al., 2019), all the taxa are new for the region.

The taxa are distributed in 7 orders (Fig. 2) and 27 families. Psathyrellaceae and Inocybaceae were found to the most crowded first two families with 16 and 7 taxa respectively. Agaricaceae, Bolbitiaceae, Incertae sedis, Morchellaceae and Strophariaceae, are the third crowded families each with 6 taxa. Then Helvellaceae and Pyronemataceae come each with 5 taxa. Two of the families (Hymenogasteraceae, Polyporaceae) comprise 4, Three of them (Pleurotaceae, Pluteaceae, Tubariaceae) comprises 3, three of them (Cortinariaceae, Entolomataceae, Pulvinulaceae) comprises 2 taxa, and the rest of the 10 families comprises 1 taxon.

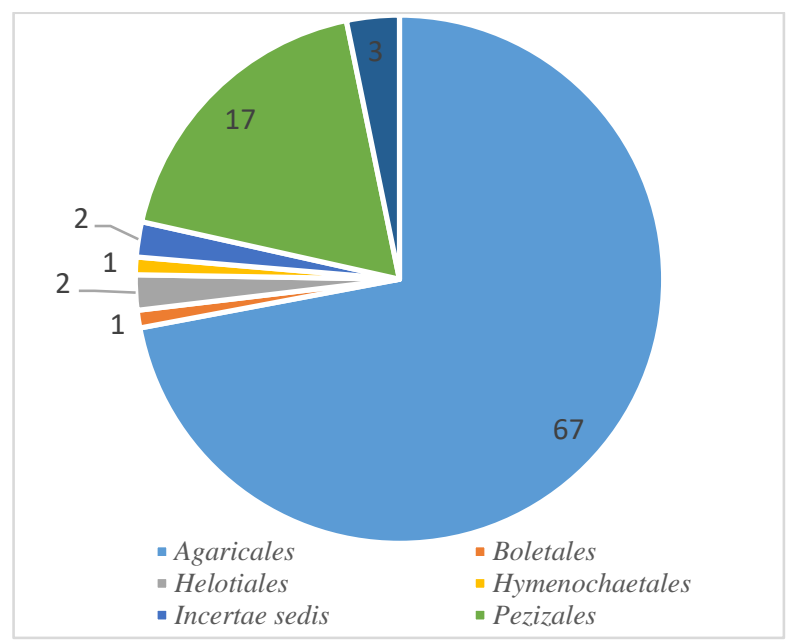

Figure 2. Ditribution of the determined taxa within orders.

The determined taxa are distributed in 49 genera. The most crowded genera are Conocybe and Psathyrella each with 6 taxa. Inocybe and Parasola were found to be the second crowded two genera each with 5 taxa. Three of of the genera (Agrocybe, Helvella, Morchella) comprise 4 taxa, five of them (Agaricus, Coprinellus, Geopora, Melanoleuca, Pleurotus) comprise 3 taxa, eight of them (Coprinopsis, Cortinarius, Entoloma, Hebeloma, Lepiota, Pluteus, Trametes, Tubaria) comprise 2 taxa, and the rest of the 29 taxa comprise one taxon.

Coprinus comatus, Coprinellus disseminatus, C. micaceus, Coprinopsis atramentaria, Psathyrella candolleana, Pleurotus ostreatus, P. eryngii, Inocybe dulcamara and Pholiota aurivella were found to be the most widespread species in the region.

Thirty six (\%38.30) of the determined taxa are edible. Among them Pleurotus eryngii is collected and consumed in all villages of the district. This mushroom is known with the Turkish name "heliz mantarı" and also has regional economic importance. Agaricus bisporus, A. campestris, A. urinascens and $P$. ostreatus are regionally known in research area and they are also collected and consumed by some locals. Forty four (\%46.81) of them are inedible and 14 (\%14.89) of them are more or less poisonous.

The taxa determined in Gürpınar district were compared with the findings of the studies carried out in neighbouring regions and some similarities were observed. These studies and the similarity percentages are given in Table 2.

Table 2. Similarity percentages of neighbouring studies with Gürpınar district.

\begin{tabular}{lccc}
\hline Neighbouring study & $\begin{array}{c}\text { \# of iden- } \\
\text { tical taxa }\end{array}$ & $\begin{array}{c}\text { Total } \\
\text { taxa }\end{array}$ & $\begin{array}{c}\text { Similarity } \\
(\%)\end{array}$ \\
\hline Bitlis (Kaya, 2001) & 17 & 60 & 28.33 \\
Ağrı (Demirel et al., 2002) & 20 & 45 & 44.44 \\
Erzurum (Demirel et al., 2003) & 17 & 114 & 14.91 \\
Malazgirt (Akçay et al., 2010) & 16 & 50 & 32.00 \\
Van (Demirel et al., 2015) & 40 & 122 & 32.79 \\
Bingöl (Uzun et al., 2017) & 16 & 112 & 14.29 \\
Şemdinli and Yüksekova (Acar & 40 & 197 & 20.30 \\
et al., 2020) & & & \\
Muradiye (Çağli and Öztürk, & 38 & 86 & 44.19 \\
2020) & & & \\
Karz Dağ1 (Sadullahoğlu and & 25 & 95 & 26.32 \\
Uzun, 2020) & & & \\
\hline
\end{tabular}

\section{Conflict of Interest}

Authors have declared no conflict of interest.

\section{Authors' Contributions}

The authors contributed equally.

\section{Acknowledgments}

The authors would like to thank Van Yüzüncü Y1l University Research Project Unit (Project No: FYL-20165213) for its financial support.

\section{References}

Acar İ, Uzun Y, Akata I (2020). Some macrofungi determined in Şemdinli and Yüksekova districts (Hakkari-Turkey). Kahramanmaraş Sütçü İmam Üniversitesi Tarım ve Doğa Dergisi 23(1): 157-167.

Adanacıoğlu N, Yıldız Ü, Erdinç O, Aykas L, Tan A, Taylan T (2016). Türkiye makromantarı genetik kaynakları I. Ege Bölgesi. Anadolu Ege Tarımsal Araştırma Enstitüsü Dergisi 26(1): 46-61.

Akçay ME, Uzun Y, Kaya A (2010). Malazgirt (Muş) yöresi makrofunguslarına katkılar. Mantar Dergisi 1(1): 14-20.

Alkan S, Kaşık G, Aktaş S 2010. Macrofungi of Derebucak district (Konya, Turkey). Turkish Journal of Botany 34(4): 335 -350. 
Bani B, Adıgüzel N (2008). Flora of an important plant area: Çatak valley I-(Çatak-Pervari) Van Turkey. Flora Mediterranea 18: $11-63$.

Breitenbach J, Kränzlin F (1984). Fungi of Switzerland, Vol.1. Lucerne: Verlag Mykologia.

Breitenbach J, Kränzlin F (1986). Fungi of Switzerland, Vol.2. Lucerne: Verlag Mykologia.

Breitenbach J, Kränzlin F (1991). Fungi of Switzerland, Vol.3. Lucerne: Verlag Mykologia.

Breitenbach J, Kränzlin F (1995). Fungi of Switzerland, Vol.4. Lucerne: Verlag Mykologia.

Breitenbach J, Kränzlin F (2000). Fungi of Switzerland, Vol.5. Lucerne: Verlag Mykologia.

Bresnsky A, Besl H (1990). A color atlas of poisonous fungi. London: Wolfe Publishing.

Buczacki S (1989). Mushrooms and toadstools of Britain and Europe. Glasgow: Harper Collins Publishers.

Çağli G, Öztürk A (2020). Macromycetes determined in Muradiye (Van) district. Anatolian Journal of Botany 4(1): 57-64.

Dähncke MR (2004). 1200 Pilze in Farbfotos. Sweden: Verlag.

Demirel K, Erdem Ö, Uzun Y, Kaya A (2010). Macrofungi of Hatila Valley National Park (Artvin-Turkey). Turkish Journal of Botany 34(5): 457-465.

Demirel K, Kaya A, Uzun Y (2003). Macrofungi of Erzurum province. Turkish Journal of Botany 27(1): 29-36.

Demirel K, Uzun Y, Akçay ME, Keleş A, Acar İ, Efe V (2015). Van yöresi makromantarlarına katkılar. Mantar Dergisi 6(2): 12 23.

Demirel K, Uzun Y, Kaya A (2002). Macrofungi of Ağr1 Province. Turkish Journal of Botany 26: 291-295.

Doğan HH, Aktaş S, Öztürk C, Kaşık G (2012). Macrofungi distribution of Cocakdere valley (Arslanköy, Mersin). Turkish Journal of Botany 36(1): 83-94.

Hausknecht A (2009). Fungi Europaei. A monograph of the genera Conocybe Fayod, Pholiotina Fayod in Europe. Italia: Massimo condusso.

Hawksworth DL, Kirk PM, Sutton BC, Pegler DN (1995). Ainsworth and Biby's dictionary of the fungi (8th Ed.). Wallingford: $\mathrm{CAB}$ International.

Index Fungorum. (2020). http://www.indexfungorum.org/names/Names.asp / [20 December 2020].

Jordan M (1995). The encyclopedia of fungi on Britain and Europe. Devon: David \& Charles Book.

Kaya A (2001). Contributions to the macrofungi flora of Bitlis province. Turkish Journal of Botany 25: 379-383.

Kaya A, Uzun Y (2018). New contributions to the Turkish Ascomycota. Turkish Journal of Botany 42: 644-652.

Keleş A, Şelem E (2017). Türkiye mikobiyotası için yeni bir kayıt (Trichophaea pseudogregaria (Rick) Boud.). Yüzüncü Yıl Üniversitesi Fen Bilimleri Enstitüsü Dergisi 22(2): 142-145.

Kuo M, Methven SA (2014). Mushrooms of the Midwest. Urbana: University of Illinois Press.

Lukić M (2008). The distribution and diversity of Boletus genus in central Serbia. Kragujevac Journal of Science 31: 59-68.

Moser M (1983). Keys to agarics and boleti (Polyporales, Boletales, Agaricales, Russulales). Stuttgart: Gustav Fischer Verlag.

Pegler DN, Læssøe T, Spooner BM (1995). British Puffballs, Earthstars, and Stinkhorns. Kew: Royal Botanic Gardens.

Phillips R (1981). Mushrooms and other fungi of Great Britain, Europe. London: Pan Books.

Sadullahoğlu C, Uzun Y (2020). Karz Dağı (Tatvan-Bitlis) ve çevresinde belirlenen makrofunguslar. Mantar Dergisi 11(1): 1-11.

Sesli E, Denchev CM (2014). Checklists of the myxomycetes, larger ascomycetes, and larger basidiomycetes in Turkey. 6th edn. Mycotaxon Checklists Online (http,//www.mycotaxon.com/resources/checklists/sesli-v106-checklist.pdf): 1-136.

Süfer Ö, Bozok F, Demir HG (2016). Usage of edible mushrooms in various food products. Turkish Journal of Agriculture-Food Science and Technology 4(3): 144-149.

Şelem E, Keleş A, Acar İ, Demirel K (2019). Edible macrofungi determined in Gürpınar (Van) district. Anatolian Journal of Botany 3(1): 7-12.

Türkekul İ, Iş̧1k H (2016). Bozatalan (Tokat) Yöresi Makrofungusları. Kafkas Üniversitesi Fen Bilimleri Enstitüsü Dergisi 9(1): 511.

Uzun Y (2010). Macrofungal diversity of Ardahan and Iğdır province. International Journal of Botany 6(1): 11-20.

Uzun Y, Acar İ, Akçay ME, Kaya A (2017). Contributions to the macrofungi of Bingöl, Turkey. Turkish Journal of Botany 41(5): 516-534.

Yıldız MS, Türkekul İ, Işı1k H (2019). Macrofungal Biodiversity of Pazar (Tokat) District. Bitlis Eren University Journal of Science 8(2): 387-395. 